\title{
Peripheral perfusion index as a predictor of failed weaning from mechanical ventilation
}

\author{
Ahmed Lotfy ${ }^{1} \cdot$ Ahmed Hasanin $^{1}$ (i) $\cdot$ Mahitab Rashad $^{1} \cdot$ Maha Mostafa $^{1} \cdot$ Dalia Saad $^{1} \cdot$ Mohamed Mahmoud $^{1}$. \\ Walid Hamimy ${ }^{1} \cdot$ Ahmed Z. Fouad $^{1}$
}

Received: 22 September 2019 / Accepted: 2 February 2020 / Published online: 8 February 2020

(c) Springer Nature B.V. 2020

\begin{abstract}
We hypothesized that impairment of peripheral perfusion index (PPI) during spontaneous breathing trial (SBT) might be predictive of weaning failure. We included 44 consecutive, adult, patients, who were scheduled for weaning after at least $48 \mathrm{~h}$ of invasive mechanical ventilation in this prospective observational study. Weaning failure was defined as failed SBT or reintubation within $48 \mathrm{~h}$ of extubation. PPI readings were obtained before initiation of the SBT, and every 5 min till the end of the SBT. PPI ratio was calculated at every time point as: PPI value/ baseline PPI. The primary outcome was the accuracy of PPI ratio at the end of the SBT in detecting failed weaning. Forty-three patients were available for the final analysis. Eighteen patients (42\%) were considered failed weaning. PPI ratio was higher in patients with successful weaning compared to patients with failed weaning during the last $15 \mathrm{~min}$ of the SBT. PPI ratio at the end of SBT was higher in patients with successful weaning compared to patients with failed weaning. PPI ratio at the end of SBT had good predictive ability for weaning failure \{area under receiver operating characteristic curve (95\% confidence interval): $0.833(0.688-0.929)$, cutoff value $\leq 1.41\}$. The change in PPI during SBT is an independent predictor for re-intubation. PPI could be a useful tool for monitoring the patient response to SBT. Patients with successful weaning showed higher augmentation of PPI during the SBT compared to re-intubated patients. Failure of augmenting the PPI by $41 \%$ at the end of SBT could predict re-intubation with negative predictive value of $95 \%$. Clinical trial identifier: NCT03974568. https://clinicaltrials.gov/ct2/show/NCT03 974568 ?term $=$ ahmed + hasanin $\&$ draw $=3 \&$ rank $=17$
\end{abstract}

Keywords Peripheral perfusion index $\cdot$ Weaning $\cdot$ Mechanical ventilation $\cdot$ Critically ill

\section{Background}

Weaning difficulty is experienced in nearly $30 \%$ of critically ill patients [1,2]. The decision to extubate mechanically ventilated critically ill patients should aim to avoid both the risk

The study was conducted in Cairo University hospitals

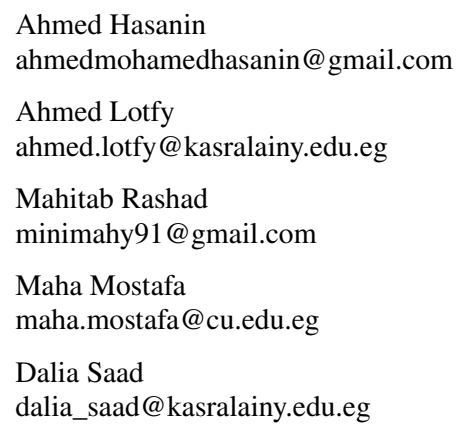

of premature liberation from mechanical ventilation which is associated with poor outcome [2]; and the risk of delayed extubation which increases the complications of prolonged mechanical ventilation [1].

Despite the various weaning indices which are recommended in the current guidelines [3], the ideal index for prediction of weaning success is not present yet $[1,4]$.

\author{
Mohamed Mahmoud \\ mohamed_mahmoud@kasralainy.edu.eg \\ Walid Hamimy \\ hamimy2002@gmail.com \\ Ahmed Z. Fouad \\ a_zaghloul2000@yahoo.com \\ 1 Department of Anesthesia and Critical Care Medicine, \\ Faculty of Medicine, Cairo University, 01 Elsarayah Street, \\ Elmanyal, Cairo 11559, Egypt
}


The most acceptable parameter for evaluation of eligibility for weaning is performing a spontaneous breathing trial (SBT). Spontaneous breathing trial is usually associated with increased oxygen demands [5] which is sometimes not matched with equivalent increase in oxygen delivery [5]. Diversion of blood flow towards respiratory muscles during the SBT impairs oxygen delivery to other tissues $[6,7]$. Impairment of various indices for peripheral tissue perfusion was found to be associated with higher risk of failed extubation $[8,9]$. Hence, monitoring of perfusion indices was hypothesized to be a useful marker for early detection of failed weaning $[8,9]$; however, the previously used indices are relatively invasive.

Peripheral perfusion index (PPI) is a simple non-invasive measure that reflects the ratio between pulsatile and nonpulsatile portions of peripheral circulation [10]. We hypothesized that impairment of PPI during SBT might be predictive of weaning failure. The aim of this work was to evaluate the possible association between PPI and success of SBT.

\section{Methods}

This prospective observational study was carried out in the surgical intensive care unit (ICU), trauma and emergency hospital, Cairo University Hospital, from June 2019 to September 2019 after being approved by the research ethics committee. The study was registered at clinicaltrials. gov registry system (Identifier: NCT03974568). Informed consent was obtained from patients' next-of-kin prior to the enrollment in the study. We consecutively included adult patients who were mechanically ventilated for more than $48 \mathrm{~h}$.

Patients aged below 18 years, patients with peripheral vascular disease, patients on vasopressors, and those with hand injuries that preclude the application of the device probe were excluded.

\subsection{Assessment of readiness to wean}

The decision of readiness to wean from mechanical ventilation was the responsibility of the attending intensivist according to the ICU protocol which included: (a) resolution of acute phase of disease for which patient was intubated; (b) adequate cough and absence of excessive tracheobronchial secretions; (c) adequate oxygenation: $\mathrm{PaO}_{2}$ more than $60 \mathrm{mmHg}$ with PEEP of $8 \mathrm{cmH}_{2} \mathrm{O}$ or less $\mathrm{cmH}_{2} \mathrm{O}$, and $\mathrm{FIO}_{2} \leq 0.5$; (d) respiratory rate (RR) of 30 breath/minute or less, $p \mathrm{H}$, and $\mathrm{PaCO}_{2}$ appropriate for patients' baseline respiratory status; e) stable cardiovascular status (i.e., heart rate (HR) less than 120 beats/min; systolic blood pressure (SBP) of 90-160 mmHg; and no or minimal vasopressor use, i.e., dopamine or dobutamine less than $5 \mu \mathrm{g} \mathrm{kg}^{-1} \mathrm{~min}^{-1}$ or noradrenaline less than $0.05 \mu \mathrm{g} \mathrm{kg}^{-1} \mathrm{~min}^{-1}[1,3]$.

\subsection{Spontaneous breathing trial (SBT)}

SBT was applied for 30 minutes through pressure support (PS) mode with PS of $5 \mathrm{cmH}_{2} \mathrm{O}$ and positive end expiratory pressure (PEEP) of $5 \mathrm{cmH}_{2} \mathrm{O}$; then, weaning parameters were re-assessed. The attending intensivist who was blinded to the PPI measurement was responsible for the decision of extubation.

Failed SBT or reintubation (or use of non-invasive ventilation) within $48 \mathrm{~h}$ of extubation were considered weaning failure as defined by the guidelines of international conference on weaning from mechanical ventilation [1, 2]. Failed SBT was considered when one or more of the following criteria were present: $\mathrm{RR}>35$ breath per minute, $\mathrm{SpO}_{2}$ less than $90 \%$ or $\mathrm{PaO}_{2}$ less than $60 \mathrm{mmHg}$ on fraction of inspired oxygen $\left(\mathrm{FIO}_{2}\right)$ of 0.4 , HR more than 140 beat per minute or sustained increase/decrease of heart rate $20 \%$ from the baseline reading, $\mathrm{SBP}>180 \mathrm{mmHg}$ or $<90 \mathrm{mmHg}$, obvious increase in accessory respiratory muscle activity, and facial signs of respiratory distress.

Radical-7 device pulse co-oximeter (Masimo corporation, Irvine, CA) was used for PPI monitoring via a pulse oximeter probe applied to the third or fourth digit of left hand. The probe was covered with an opaque shield to avoid any artifact caused by external light sources. PPI readings were obtained as a baseline reading before initiation of the SBT, then every $5 \mathrm{~min}$ till the end of the SBT. We calculated PPI ratio to evaluate the change in PPI during SBT. PPI was observed for $30 \mathrm{~s}$ and the average of the highest and lowest values was recorded. PPI ratio was calculated at every time point as: PPI value/ baseline PPI.

\subsection{Outcomes}

\subsubsection{Primary outcome}

The accuracy of PPI ratio at the end of the SBT in detecting failed weaning.

\subsubsection{Secondary outcomes}

PPI: measured every 5 min during the SBT.

PPI ratio every 5 min during the SBT.

Hemodynamic data: HR, SBP, and central venous pressure. Data were recorded at the baseline (before SBT) and every 5 min till the end of SBT.

Respiratory rate was recorded at the baseline (before SBT), and by the end of the SBT. Respiratory rate ratio was calculated as: respiratory rate by the end of the SBT / respiratory rate at the baseline. 
Rapid shallow breathing index (RSBI) was recorded at the baseline (before SBT), and by the end of the SBT. RSBI was calculated by dividing respiratory rate / tidal volume. RSBI ratio was calculated as: RSBI by the end of the SBT / RSBI at the baseline.

Other data included severity scores, $\mathrm{PaO}_{2} / \mathrm{F}_{\mathrm{I}} \mathrm{O}_{2}(\mathrm{P} / \mathrm{F}$ ratio), $p \mathrm{H}, \mathrm{PaCO}_{2}$, serum $\mathrm{HCO}_{3}$, serum lactate, fluid balance, urine output, and days of mechanical ventilation.

\subsection{Statistical analysis and sample size calculation}

We calculated our sample size using MedCalc version 12.1.4.0 (MedCalc Software bvba, Mariakerke, Belgium) to detect area under receiver operating characteristic (AUROC) curve of at least 0.75 for PPI to predict weaning failure and the null hypothesis of AUROC was 0.5. The minimum number which was needed to have a study power of $80 \%$ and alpha error of 0.05 was 42 patients with at least 14 positive (weaning failure) cases.

Data were presented as mean \pm SD or median and interquartile range for continuous variables and as absolute or relative frequencies for categorical variables. Unpaired Student's $t$ test or Mann-Whitney $U$ test were used to compare continuous variables, and chi-squared test, or Fisher's exact test were used to compare categorical variables. For comparison of successful weaning group, failed SBT group and reintubation group: normally distributed data were analyzed using one-way analysis of variance (ANOVA) with post-hoc pairwise comparisons using the Tukey test; skewed data were analyzed using Kruskal-Wallis test (oneway ANOVA for ranks). Repeated measures were analyzed using ANOVA for repeated measures with Bonferroni correction. Multivariate regression model was created including variables with $P$ values of less than 0.2 in the univariate analysis. Variables with $P$ values $\leq 0.05$ in the multivariate analysis were considered statistically significant. Receiver operating characteristic curves were constructed and the area under the curve was calculated. MedCalc version 12.1.4.0 (MedCalc Software bvba, Mariakerke, Belgium) generated values with the highest sensitivity and specificity (Youden index). The level of significance was set at $\mathrm{P}<0.05$.

\section{Results}

Forty-eight patients were available for screening. Four patients were excluded for being tracheostomized and 1 patient was excluded due to poor PPI signal. Forty-three patients were available for final analysis ( 25 patients with successful weaning and 18 patients with failed weaning). Seven patients of the failed weaning group had a failed SBT; whereas, 11 patients were successfully extubated and were then re-intubated within $48 \mathrm{~h}$ (Fig. 1).

Patients with successful weaning had lower age compared to patients with failed weaning $(40 \pm 17$ years versus $57 \pm 13$ years, $\mathrm{P}=0.001)$. Other demographic data and baseline characteristics were comparable between the successful weaning group and failed weaning group. Most of the respiratory parameters $\left(p \mathrm{H}, \mathrm{PaCO}_{2}, \mathrm{HCO}_{3}\right.$, and $\mathrm{P} / \mathrm{F}$ ratio $)$ and hemodynamic variables (central venous pressure, SBP and $\mathrm{HR}$ ) were comparable between the successful weaning and the failed weaning groups except for the RR and the RSBI which were lower in patients with successful weaning compared to patients with failed weaning (Tables 1,2 ).

PPI was comparable between the successful weaning group and the failed weaning group at the all readings.
Fig. 1 Flow chart showing patient recruitment. SBT spontaneous breathing trial

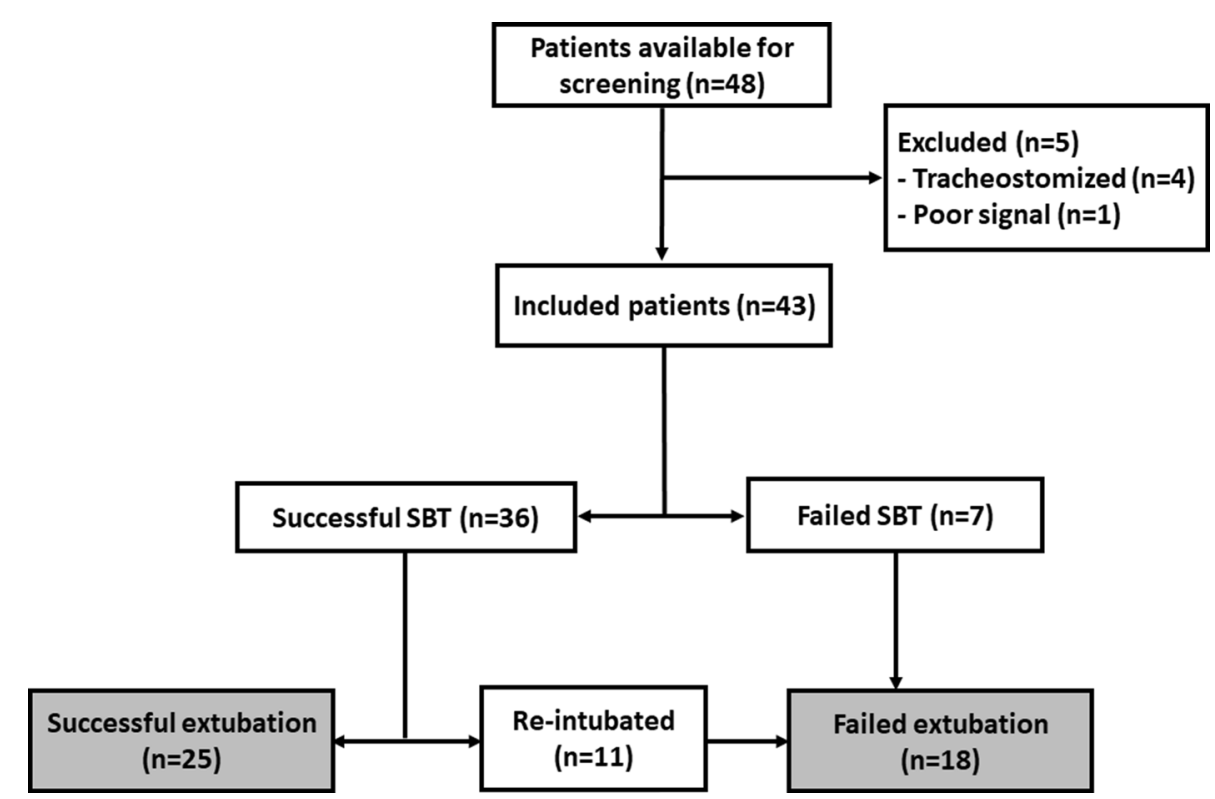


Table 1 Demographic data and baseline characteristics

\begin{tabular}{|c|c|c|c|}
\hline & $\begin{array}{l}\text { Successful weaning } \\
(\mathrm{n}=25)\end{array}$ & Failed weaning $(n=18)$ & $\mathrm{P}$ value \\
\hline Age (years) & $40 \pm 17$ & $57 \pm 13$ & 0.001 \\
\hline Weight (kg) & $78 \pm 7$ & $79 \pm 8$ & 0.67 \\
\hline Male gender (\%) & $12(46 \%)$ & $13(68 \%)$ & 0.26 \\
\hline APACHE score & $20.6 \pm 8$ & $20.9 \pm 5$ & 0.89 \\
\hline \multicolumn{4}{|l|}{ Number of previous trials (\%) } \\
\hline 0 & $20(80)$ & $14(78)$ & 0.482 \\
\hline 1 & $5(20)$ & $3(19)$ & \\
\hline 3 & $0(0)$ & $1(2)$ & \\
\hline GCS $(\%)$ & & & 1 \\
\hline 13 & $1(4)$ & $1(6)$ & \\
\hline 15 & $24(96)$ & $17(94)$ & \\
\hline ARDS (\%) & $2(8)$ & $0(0)$ & 0.502 \\
\hline COPD $(\%)$ & $1(4)$ & $3(17)$ & 0.293 \\
\hline Renal impairment (\%) & $12(48)$ & $8(44)$ & 1 \\
\hline Septic shock (\%) & $14(56)$ & $9(50)$ & 0.763 \\
\hline Post laparotomy (\%) & $14(56)$ & $10(56)$ & 1 \\
\hline Blood hemoglobin (g/dL) & $11.2 \pm 2.3$ & $10.3 \pm 2.2$ & 0.23 \\
\hline Serum albumin $(\mathrm{g} / \mathrm{dL})$ & $2.2 \pm 0.5$ & $2.1 \pm 0.5$ & 0.64 \\
\hline Duration of MV (days) & $2.9 \pm 1.9$ & $2.5 \pm 1.1$ & 0.46 \\
\hline $\mathrm{NE}$ infusion rate (mcg/kg/min) & $0(0-0.12)$ & $0(0-0.18)$ & 0.935 \\
\hline Fluid input in pre-SBT day (mL) & $2590 \pm 983$ & $3232 \pm 1107$ & 0.052 \\
\hline Urine output in pre-SBT day $(\mathrm{mL})$ & $2530 \pm 1394$ & $2977 \pm 926$ & 0.24 \\
\hline Net fluid balance in pre-SBT day $(\mathrm{mL})$ & $114 \pm 1045$ & $229 \pm 815$ & 0.67 \\
\hline
\end{tabular}

Data are presented as mean $\pm \mathrm{SD}$, median (quartiles), and frequency (\%)

$A P A C H E$ acute physiology and chronic health evaluation, $M V$ mechanical ventilation, $N E$ norepinephrine, $S B T$ spontaneous breathing trial

\begin{tabular}{llll}
\hline & $\begin{array}{l}\text { Successful weaning } \\
(\mathrm{n}=25)\end{array}$ & Failed weaning $(\mathrm{n}=18)$ & P value \\
\hline $\mathrm{P} / \mathrm{F}$ ratio & $345 \pm 106$ & $280 \pm 107$ & 0.054 \\
$\mathrm{PaCO}_{2}(\mathrm{mmHg})$ & $30.2 \pm 6$ & $31.8 \pm 5$ & 0.37 \\
$p \mathrm{H}$ & $7.44 \pm 0.1$ & $7.46 \pm 0.07$ & 0.4 \\
$\mathrm{HCO} 3(\mathrm{mmol} / \mathrm{L})$ & $20.7(19-26.2)$ & $23.5(20.1-27.2)$ & 0.17 \\
$\mathrm{Lactate}(\mathrm{mmol} / \mathrm{L})$ & $1.35(0.93-1.7)$ & $1.4(1.1-1.8)$ & 0.4 \\
$\mathrm{CVP}\left(\mathrm{cmH} \mathrm{H}_{2} \mathrm{O}\right)$ & $7.8 \pm 3.6$ & $8.9 \pm 3.4$ & 0.36 \\
$\mathrm{SBP}(\mathrm{mmHg})$ & $128 \pm 16$ & $128 \pm 19$ & 0.936 \\
Heart rate $(\mathrm{bpm})$ & $97 \pm 18$ & $98 \pm 15$ & 0.857 \\
Compliance $\left(\mathrm{mL} \cdot \mathrm{H}_{2} \mathrm{O}^{-1}\right)$ & $87 \pm 26$ & $72 \pm 26$ & 0.1 \\
\hline
\end{tabular}

Data are presented as mean standard deviation, and median (quartiles)

$C V P$ central venous pressure, $\mathrm{P} / \mathrm{F}$ ratio: $\mathrm{PO}_{2} /$ fraction of inspired oxygen, $R S B I$ rapid shallow breathing index, $S B P$ systolic blood pressure, $S B T$ spontaneous breathing trial
Table 2 Blood gases, respiratory mechanics, and hemodynamic variables before initiation of spontaneous breathing trial
The successful weaning group had lower baseline RR and RSBI compared to the failed SBT group, whereas, both variables were comparable between successful weaning group and re-intubation group. The PPI ratio after $15 \mathrm{~min}$ was lower in the failed SBT group compared to the successful 


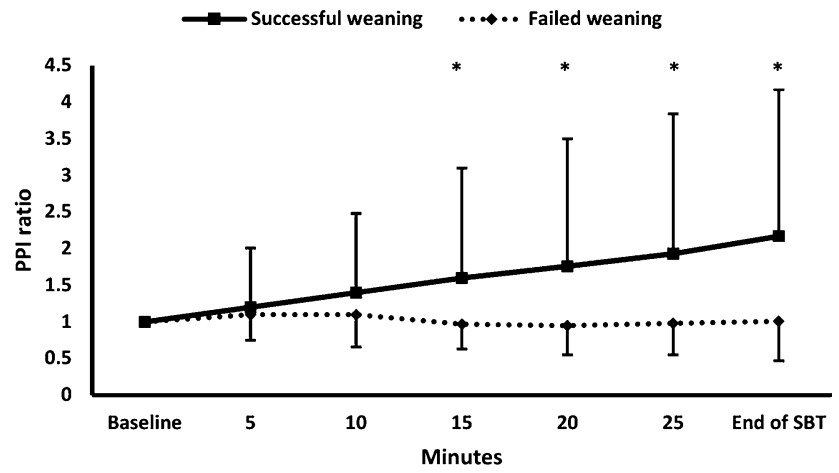

Fig. 2 Time course of PPI during the SBT. Markers are means, error bars are standard deviations. All values are presented as proportions from the baseline reading. PPI peripheral perfusion index. SBT spontaneous breathing trial. *denotes statistical significance between both groups

weaning group ( $P$ value: 0.004$)$. The PPI ratio after $30 \mathrm{~min}$ was lower in each of failed SBT group and re-intubation group compared to the successful weaning group. ( $P$ values: 0.001 and 0.049 , respectively). (Table 3 ).

Multivariate analysis revealed that higher age and lower PPI ratio after $30 \mathrm{~min}$ were the only independent risk factor for reintubation (odds ratio [95\% CI]: 1.2 [1-1.5] and 0.02 $[<0.001-0.8]$ respectively).

AUROC curve for predicting weaning failure was highest for PPI ratio after $30 \mathrm{~min}$ \{AUROC $(95 \% \mathrm{CI})$ : $0.83(0.69-0.93)$, cutoff value $\leq 1.41\}$, followed by baseline respiratory rate $\{$ AUROC $(95 \% \mathrm{CI})$ : 0.80(0.65-0.91), cutoff value $>23\}$, and baseline RSBI \{AUROC $(95 \% \mathrm{CI})$ : 0.76(0.61-0.88), cutoff value $>39.5\}$ (Table 4).

AUROC curve for predicting re-intubation was highest for PPI ratio after $30 \mathrm{~min}$ \{ AUROC (95\% CI): 0.78 (0.61-0.9), cutoff value $\leq 1.41\}$, followed by respiratory rate post SBT \{ AUROC (95\% CI): 0.75 (0.58-0.88), cutoff value $>22$ \}, and baseline respiratory rate $\{$ AUROC $(95 \% \mathrm{CI}): 0.73$ (0.56-0.87), cutoff value $>19\}$. AUROC $(95 \%$ CI) of RSBI at baseline and post SBT for predicting re-intubation were $0.71(0.53-0.85)$, cutoff value $>39.5$ and $0.71(0.54-0.85)$, cutoff value $>42.5$, respectively (Table 5 ).

\section{Discussion}

In our study, we evaluated the change in PPI during SBT. We reported an association between the change in PPI during SBT and the weaning outcome. Patients with successful
Table 3 PPI, respiratory variables and hemodynamic variables before and after SBT

\begin{tabular}{|c|c|c|c|c|}
\hline & $\begin{array}{l}\text { Successful wean- } \\
\text { ing }(\mathrm{n}=25)\end{array}$ & Failed SBT $(n=7)$ & Reintubation $(n=11)$ & $P$ value \\
\hline \multicolumn{5}{|l|}{$\mathrm{SBP}(\mathrm{mmHg})$} \\
\hline Before SBT & $129 \pm 16$ & $134 \pm 24$ & $124 \pm 15$ & 0.596 \\
\hline By the end of SBT & $127 \pm 18$ & $119 \pm 34$ & $124 \pm 13$ & 0.647 \\
\hline \multicolumn{5}{|c|}{ Heart rate (beat per minute) } \\
\hline Before SBT & $97 \pm 18$ & $110 \pm 30$ & $96 \pm 14$ & 0.311 \\
\hline By the end of SBT & $98 \pm 16$ & $106 \pm 27$ & $98 \pm 14$ & 0.576 \\
\hline \multicolumn{5}{|l|}{ Respiratory rate/min } \\
\hline Before SBT & $11(16,23)$ & $28(25,34)^{*}$ & $24(20,27)$ & 0.002 \\
\hline By the end of SBT & $19(15,24)$ & $30(24,34)^{*}$ & $25(23,26)$ & 0.003 \\
\hline \multicolumn{5}{|l|}{ RSBI } \\
\hline Before SBT & $41(27,68)$ & $83(49,100)^{*}$ & $67(44,74)$ & 0.011 \\
\hline By the end of SBT & $36(26,55)$ & $85(29,99)^{*}$ & $58(40,71)$ & 0.007 \\
\hline \multicolumn{5}{|l|}{ PPI } \\
\hline Before SBT & $2.4(0.74,3.5)$ & $6.7(1.1,13)$ & $1.3(0.74,7.1)$ & 0.581 \\
\hline At $15 \mathrm{~min}$ & $3(0.9,5)$ & $3(0.3,8.8)$ & $2.3(0.7,8.2)$ & 0.986 \\
\hline By the end of SBT & $3.5(1.6,6.1)$ & $3.8(1.4,7.7)$ & $2.1(0.67,7.4)$ & 0.674 \\
\hline \multicolumn{5}{|l|}{ PPI ratio } \\
\hline At $15 \mathrm{~min}$ & $1.1(0.99,1.7)$ & $0.7(0.65,0.8)^{*}$ & $1(0.8,1.3)$ & 0.009 \\
\hline At the end of SBT & $1.8(1.3,2.3)$ & $0.7(0.5,1)^{*}$ & $1(0.9,1.4)^{*}$ & $<0.001$ \\
\hline Respiratory rate ratio & $1(0.9,1.1)$ & $1(0.9,1.1)$ & $1.1(1,1.2)$ & 0.336 \\
\hline RSBI ratio & $1.1(0.99,1.2)$ & $1(0.95,1.1)$ & $1(0.98,1.4)$ & 0.346 \\
\hline
\end{tabular}

Data are presented as mean \pm standard deviation and median (quartiles)

$P P I$ peripheral perfusion index, RSBI rapid shallow breathing index, SBT spontaneous breathing trial. *Denotes significance in relation to the successful weaning group 
Table 4 Receiver operating characteristic properties for prediction of weaning failure

$\begin{array}{lllll}\text { AUROC }(95 \% \mathrm{CI}) & \text { Sensitivity } & \text { Specificity }(\%) & \text { PPV }(\%) & \text { NPV }(\%)\end{array}$

$(\%)$

\begin{tabular}{lllrrrr}
\hline Respiratory rate (baseline) & $0.80(0.65-0.91)^{*}$ & 65 & 84 & 73 & 78 & $>23$ \\
RSBI (baseline) & $0.76(0.61-0.88)^{*}$ & 88 & 56 & 58 & 88 & $>39.5$ \\
PPI (baseline) & $0.57(0.41-0.72)$ & 33 & 100 & 100 & 67 & $>6.1$ \\
PPI ratio (15 min) & $0.71(0.55-0.84)^{*}$ & 56 & 84 & 71 & 72 & $\leq 0.88$ \\
PPI ratio (30 min) & $0.83(0.69-0.93)^{*}$ & 94 & 72 & 71 & 95 & $\leq 1.41$ \\
\hline
\end{tabular}

*Denotes statistical significance $(\mathrm{P}$ value $<0.05)$. AUROC Area under receiver operating characteristic curve, $N P V$ negative predictive value, $P P I$ peripheral perfusion index, $P P V$ positive predictive value. $R S B I$ rapid shallow breathing index

Table 5 Receiver operating characteristic properties for prediction of re-intubation

\begin{tabular}{llllllc}
\hline & AUROC (95\% CI) & $\begin{array}{l}\text { Sensi- } \\
\text { tivity } \\
(\%)\end{array}$ & $\begin{array}{l}\text { Speci- } \\
\text { ficity } \\
(\%)\end{array}$ & PPV (\%) & NPV (\%) & Cutoff value \\
\hline Respiratory rate (baseline) & $0.73(0.56-0.87)^{*}$ & 82 & 60 & 47 & 88 & $>19$ \\
Respiratory rate (30 min) & $0.75(0.58-0.88)^{*}$ & 82 & 72 & 56 & 90 & $>22$ \\
Respiratory rate ratio & $0.64(0.46-0.79)$ & 91 & 48 & 44 & 92 & $>0.95$ \\
RSBI (baseline) & $0.71(0.53-0.85)^{*}$ & 82 & 56 & 45 & 88 & $>39.5$ \\
RSBI (30 min) & $0.71(0.54-0.85)$ & 82 & 60 & 47 & 88 & $>42.5$ \\
RSBI ratio & $0.52(0.35-0.69)$ & 64 & 68 & 47 & 81 & $>1.06$ \\
PPI (baseline) & $0.54(0.37-0.71)$ & 36 & 92 & 67 & 74 & $>4.4$ \\
PPI ratio (15 min) & $0.63(0.45-0.78)$ & 82 & 44 & 39 & 84 & $\leq 1.34$ \\
PPI ratio (30 min) & $0.78(0.61-0.9)^{*}$ & 91 & 72 & 59 & 95 & $\leq 1.41$ \\
\hline
\end{tabular}

*Denotes statistical significance $(\mathrm{P}$ value $<0.05)$. AUROC Area under receiver operating characteristic curve, $C I$ confidence interval, $N P V$ negative predictive value, $P P I$ peripheral perfusion index, $P P V$ positive predictive value. $R S B I$ rapid shallow breathing index. "denotes statistical significance weaning augmented the PPI by $>41 \%$ during SBT; whilst, patients with failed weaning were unable to augment the PPI during SBT to the same extent. Thus, serial measurement of PPI could predict weaning failure.

Switching the patient from assisted to spontaneous ventilation increases preload, afterload, and catecholamine release [11]; thus, it is usually associated with increased cardiac output [12] which would consequently increase the PPI. PPI reflects the adequacy of blood flow in the peripheral circulation. PPI decreases in hypovolemia [13]; thus, it is considered an indirect index for the adequacy of cardiac output. PPI was recently used as a surrogate of the cardiac output during resuscitation of shocked patients [14]. This relation between the PPI and the cardiac output could explain the change in the PPI during the SBT. In patients with failed SBT, there is an increased oxygen demand which is not compensated with increased oxygen delivery with increased blood flow to the respiratory muscles on the expense of peripheral tissues. Hence, patients who fail to augment the PPI during the SBT are more likely to fail during the weaning trial.

In line with our explanation to the PPI change during SBT, Tanios et al. had reported augmentation of the cardiac output during SBT in all patients which is more evident in patients with successful weaning [12]. Frazier et al. had reported that the cardiac output increased significantly during the SBT in patients with successful weaning; whilst, no change was reported in the cardiac output in patients with failed weaning [11].

SBT is a stressful event which is used to evaluate the patient's ability to maintain breathing without ventilator support. Switching the patient from assisted to spontaneous ventilation is usually associated with increased oxygen demands [5], which might not be matched with a proportional increase in the oxygen delivery. During the SBT, blood flow is diverted towards respiratory muscles; this impairs the oxygen delivery to other tissues $[6,7]$. Subsequently, various indices for peripheral tissue perfusion were reported to be impaired in patients with failed extubation trials such as serum lactate [8], central venous oxygen saturation [9], thenar oxygen saturation, [15], mottling score, and knee oxygen saturation [16]. Most of these indices are either invasive or expensive. PPI is characterized by being non-invasive, real time, and simple variable [10] which is easily measured through a special pulse oximeter probe. 
PPI is characterized by high skewness and variability in its baseline readings [10]. Thus, comparison of PPI values is usually performed through PPI ratio, which represents the change of PPI before and after the intervention. We evaluated the change of PPI in our patients during the SBT by comparing serial PPI ratio in each time point. Using PPI ratio was previously reported by our group in evaluating the change in PPI after various interventions [17, 18].

The difference in PPI values between patients with successful weaning and patients with failed weaning was demonstrated through the change in its values during the SBT, and not in the baseline values. This indicates that assessment of the resting PPI before undergoing the SBT would not identify the high-risk patients.

Discontinuation of mechanical ventilation is an important challenging decision in intensive care medicine. Failure of weaning and re-intubation is associated with poor patient outcomes [2]. Thus, proper evaluation of patient readiness for weaning would improve patient outcomes and avoid unwarranted re-intubation. Using PPI for predicting weaning outcome could be a complementary tool that improve the decision of patient extubation and avoid the risks of re-intubation. We demonstrated that PPI starts to differentiate between patients with successful weaning and patients with failed weaning $15 \mathrm{~min}$ after the beginning of the SBT. This finding would allow early identification of the weaning outcome which would alert the intensivist to abort the SBT to avoid unwarranted deterioration of the vital signs in some patients.

Our study had some limitations: (1) It is a single center study. (2) Due to the nature of our surgical ICU, all participants in our study are either postoperative emergency patients or trauma patients. (3) We could not perform subgroup analysis for cardiac patients because our sample size was not designed for this objective. We recommend future studies that evaluate PPI changes during weaning of special subgroups of patients such as medical patients and cardiac patients. Future studies would also confirm the cut-off values for predicting weaning failure and combine the PPI with other traditional indices such as RSBI and $\mathrm{P} / \mathrm{F}$ ratio.

In conclusion, the change in PPI during SBT is an independent predictor for re-intubation. PPI could be a useful tool for monitoring patients during SBT. Patients with successful weaning showed higher augmentation of the PPI during the SBT compared to patients with failed weaning. Failure of augmenting PPI by $41 \%$ at the end of SBT could predict re-intubation with NPV of $95 \%$.

Funding This research did not receive any specific grant from funding agencies in the public, commercial, or not-for-profit sectors.
Data availability The data that support the findings of this study are available from Cairo university hospitals; however, they are not publicly available. Data are however available from the authors upon reasonable request after permission of Cairo university.

\section{Compliance with ethical standards}

Conflict of interest The authors declare that they have no conflict of interest with this work.

Ethical approval Our paper was approved by the Research Ethics Committee, Faculty of medicine, Cairo University (N-159-2018), on 16 March 2019.

\section{References}

1. Peñuelas Ó, Thille AW, Esteban A. Discontinuation of ventilatory support. Curr Opin Crit Care. 2015;21:74-81. https://doi. org/10.1097/MCC.0000000000000169.

2. Boles J-M, Bion J, Connors A, Herridge M, Marsh B, Melot C, Pearl R, Silverman H, Stanchina M, Vieillard-Baron A, Welte T. Weaning from mechanical ventilation. Eur Respir J. 2007;29:1033-56. https://doi.org/10.1183/09031936.00010206.

3. Girard TD, Alhazzani W, Kress JP, Ouellette DR, Schmidt GA, Truwit JD, Burns SM, Epstein SK, Esteban A, Fan E, Ferrer M, Fraser GL, Gong MN, Hough CL, Mehta S, Nanchal R, Patel S, Pawlik AJ, Schweickert WD, Sessler CN, Strøm T, Wilson KC, Morris PE. An official american thoracic society/american college of chest physicians clinical practice guideline: liberation from mechanical ventilation in critically ill adults rehabilitation protocols, ventilator liberation protocols, and cuff leak tests. Am J Respir Crit Care Med. 2017;195:120-33. https://doi.org/10.1164/ rccm.201610-2075ST.

4. El-Khatib MF, Bou-Khalil P. Clinical review: liberation from mechanical ventilation. Crit Care. 2008;12:1-11. https://doi. org/10.1186/cc6959.

5. Field S, Kelly SM, Macklem PT. The oxygen cost of breathing in patients with cardiorespiratory disease. Am Rev Respir Dis. 1982;126:9-13. https://doi.org/10.1164/arrd.1982.126.1.9.

6. Magder S, Erian R, Roussos C. Respiratory muscle blood flow in oleic acid-induced pulmonary edema. J Appl Physiol. 1986;60:1849-56. https://doi.org/10.1152/jappl.1986.60.6.1849.

7. Pang LM, Kim YJ, Bazzy AR. Blood flow to respiratory muscles and major organs during inspiratory flow resistive loads. J Appl Physiol. 1993;74:428-34. https://doi.org/10.1152/jappl 1993.74.1.428.

8. Zakynthinos S, Routsi C, Vassilakopoulos T, Kaltsas P, Zakynthinos E, Kazi D, Roussos C. Differential cardiovascular responses during weaning failure: effects on tissue oxygenation and lactate. Intensive Care Med. 2005;31:1634-42. https://doi.org/10.1007/ s00134-005-2699-9.

9. Teixeira C, da Silva NB, Savi A, Vieira SRR, Nasi LA, Friedman G, Oliveira RP, Cremonese RV, Tonietto TF, Bressel MAB, Maccari JG, Wickert R, Borges LG. Central venous saturation is a predictor of reintubation in difficult-to-wean patients. Crit Care Med. 2010;38:491-6. https://doi.org/10.1097/CCM.0b013e3181 bc81ec.

10. Hasanin A, Mukhtar A, Nassar H. Perfusion indices revisited J Intensive Care. 2017;5:24. https://doi.org/10.1186/s4056 0-017-0220-5.

11. Frazier SK, Stone KS, Moser D, Schlanger R, Carle C, Pender L, Widener J, Brom H. Hemodynamic changes during 
discontinuation of mechanical ventilation in medical intensive care unit patients. Am J Crit Care. 2006;15:580-93. Quiz 594

12. Tanios M, Epstein S, Sauser S, Chi A. Noninvasive monitoring of cardiac output during weaning from mechanical ventilation: a pilot study. Am J Crit Care. 2016;25:257-65. https://doi. org/10.4037/ajcc2016921.

13. Høiseth LØ, Hisdal J, Hoff IE, Hagen OA, Landsverk SA, Kirkebøen KA. Tissue oxygen saturation and finger perfusion index in central hypovolemia: influence of pain. Crit Care Med. 2015;43:747-56. https://doi.org/10.1097/CCM.000000000000076 6.

14. Beurton A, Teboul J-L, Gavelli F, Gonzalez FA, Girotto V, Galarza L, Anguel N, Richard C, Monnet X. The effects of passive leg raising may be detected by the plethysmographic oxygen saturation signal in critically ill patients. Crit Care. 2019;23:19. https://doi.org/10.1186/s13054-019-2306-z.

15. Poriazi M, Kontogiorgi M, Angelopoulos E, Vasileiadis I, Tripodaki ES, Nanou V, Fassoulaki A, Nanas S, Routsi C. Changes in thenar muscle tissue oxygen saturation assessed by near-infrared spectroscopy during weaning from mechanical ventilation. Minerva Anestesiol. 2014;80:666-75.
16. Margetis D, Maury E, Boelle PY, Alves M, Galbois A, Baudel JI, Offenstadt G, Guidet B, Ait-Oufella H. Peripheral microcirculatory exploration during mechanical ventilation weaning. Minerva Anestesiol. 2014;80:1188-97.

17. Hasanin A, Mohamed SAR, El-adawy A. Evaluation of perfusion index as a tool for pain assessment in critically ill patients. J Clin Monit Comput. 2017;31:961-5. https://doi.org/10.1007/s1087 7-016-9936-3.

18. Abdelnasser A, Abdelhamid B, Elsonbaty A, Hasanin A, Rady A. Predicting successful supraclavicular brachial plexus block using pulse oximeter perfusion index. Br J Anaesth. 2017;119:276-80. https://doi.org/10.1093/bja/aex166.

Publisher's Note Springer Nature remains neutral with regard to jurisdictional claims in published maps and institutional affiliations. 